NASA Technical Memorandum 86743

NASA-TM-86743 19860001739

\title{
Water Tunnel Flow Visualization Using a Laser
}

Christine Beckner and Robert E. Curry

October 1985
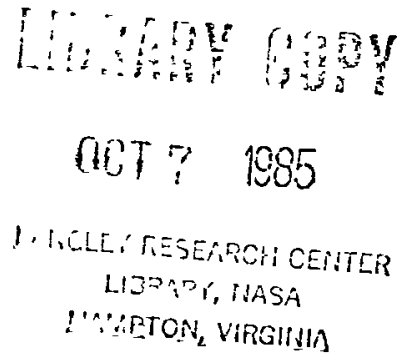
NASA Technıcal Memorandum 86743

\section{Water Tunnel Flow Visualization Using a Laser}

Christıne Beckner and Robert E Curry

Ames Research Center, Dryden Flight Research Facility, Edwards, Calıfornia

Natıonal Aeronautics and

Space Admınıstratıon

Ames Research Center

Dryden Flight Research Facility

Edwards, Calıfornı 93523 
WATER TUNNEL FLOW VISUALIZATION USING A LASER

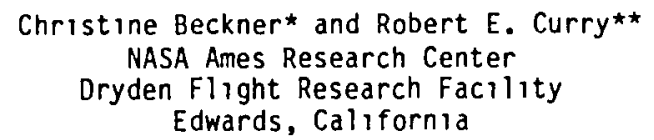

\section{Abstract}

Laser systems for flow visualization in water tunnels (similar to the vapor screen technique used in wind tunnels) can provide two-dimensional crosssectional views of complex flow fields. This parametric study documents the practical application of the laser-enhanced visualization (LEV) technique to water tunnel testing. Aspects of the study include laser power levels, flow seeding (using fiourescent dyes and embedded particulates), model preparation, and photographic techniques. The results of this study are discussed to provide potential users with basic information to aid in the design and setup of an LEV system.

\section{Introduction}

Water tunnels provide a convenient means of observing three-dimensional flow phenomena, such as vortices. A thin sheet of high-intensity light produced by a laser light source can be scanned across the model being studied (in either a vertical or a horizontal plane) to provide twodimensional cross-sectional views of the flow field. By seeding the flow with injected fluorescent dyes or embedded particulate matter, crosssectional views of the flow field can be documented photographically or by video recording. This scheme, generally similar to the vapor screen technique used in wind tunnels, 1-3 has previously been applied to water tunnels. 4 Using this flow seeding method, laser-enhanced visualization (LEV) techniques can provide a detalled qualitative analysis of internal flow patterns. The technique can be used for quantitative measurements as well.

An LEV study was undertaken using the flow visualization system (FVS) located at the Dryden Flight Research Fac1lity of the NASA Ames Research Center to address some of the practical aspects related to water tunnel applications, such as safety, flow seeding, and photographic documentation techniques. The procedures and techniques presented in this paper have produced reliable results.

\section{Experimental Equipment and Procedures}

\section{Water Tunnel}

The NASA Ames-Dryden FVS is a single-return water tunnel with a vertical test section (see Fig. 1). The test section is 0.61 by $0.41 \mathrm{~m}$ ( 24 by $16 \mathrm{in}$ ) in cross section. The walls of the test section are made of clear plexiglass, $5.1 \mathrm{~cm}$ $(2.0 \mathrm{in})$ thick. The Reynolds number at the test

\footnotetext{
*Aerospace engineerıng student trainee.

**Aerospace Engineer. Member AIAA.
}

section can be varied from 13,200 to $330,000 \mathrm{~m}^{-1}$ $\left(4000\right.$ to $\left.100,000 \mathrm{ft}^{-1}\right)$.

Compared with other ground-based flow analysis techniques, conventional operation of the water tunnel is relatively simple and inexpensive. Technical observers can be accommodated, and test plans and models can easily be modified as preliminary results are obtained. These aspects contribute to the effectiveness of the FVS for qualitative analysis.

\section{Laser System}

The first concern of the study was the identification of a safe yet effective laser output power level for routine operations. A $4-W$, class IV argon laser was used, which required a $208-V$, three-phase power supply and 7.611 ter/min (2 gal $/ \mathrm{min}$ ) of cooling water. This laser was used because of avallability and the fact that it could be operated over the desired power settings ranging from 250 to $1000 \mathrm{~mW}$ (see Table 1). (Lower power lasers requiring a $110-V$ power supply and no cooling water would have been adequate but were not readily avallable for this project.) The laser power supply was located near the water tunnel test section so that the system could be shut down 1 mmediately if a hazard arose. Other emergency means of shutting down the laser system (puling circuit breakers or turning off the cooling water) were brought to the attention of all LEV personnel.

\section{Experimental Test Setup}

Special consideration was given to the design of the equipment installation because of the poten tially hazardous laser light levels. Certain precautions were necessary to ensure personnel safety and also to prohibit extraneous light from interfering with the experiment. This detracted from the ease and simplicity of operation normally assocrated with the water tunnel. Figure 2 shows the schematic of the water tunnel test setup. The entire test area was screened of $f$, and laser warning signs and red flashing lights were placed at both entry areas. A black felt curtain enclosed the plexiglass test section to terminate the laser beam and to keep out external light. Overhead 1 amps around the test section were turned of $f$ when tests were conducted to further reduce the $1 \mathrm{ncl}-$ dence of nonlaser light. A fabric shroud 1 solated the photographic area from reflections and glare.

In order to 1 solate the laser beam during testing, a light-tight box was constructed to contain the laser head, al ignment mirrors, and other test equipment (see Fig. 3). The dimensions of the laser isolation box are 30 by 51 by $137 \mathrm{~cm}$ ( 12 by 20 by $54 \mathrm{in}$ ). The box was closed during tests except for the end directed toward the water tunnel test section. The laser head was situated 
within the box with the output aperture away from the water tunnel, this orientation allowed the beam to be folded, which provided more room for beam alignment and future expansion of the optical capability.

To align the beam and mirrors, the laser was operated at a low power level (approximately $200 \mathrm{mh}$ output), and protective eyewear (which blocks laser light frequencies) was worn. The beam causes some local flourescence on a target held in its path, this fluorescence can be seen with safety glasses on, and alignment can be affected. The beam was directed toward the front of the box and passed through an iris diaphragm, which narrowed the beam. It was then projected through a $0.476-\mathrm{cm}$ (0.1875-1n) diameter glass rod, which served as a cylindrical lens to spread the beam into a sheet of light. The final mirror projected the light sheet into the water tunnel test section and onto the model. Movement of this mirror allowed the light sheet to be scanned across the test section. The thickness of the light sheet in the test section was about $1.6 \mathrm{~mm}(0.063 \mathrm{ln})$. After this alignment was complete the box was sealed. With the output emitted directly into the water tunnel, the potential for eye injury was greatly reduced.

In a later modification, the stand supporting the laser isolation box was modified so that the laser light sheet could be scanned across the test section by moving the box (horizontally or vertically). Using this configuration it was possible to project the laser beam through the cylindrical lens directly into the water tunnel test section without the use of mirrors.

Streamwise flow was observed by projecting the laser beam through the cylindrical lens mounted horizontally to produce a vertical sheet of light. In a similar manner, the cylindrical lens was mounted vertically to produce a horizontal sheet of light. However, special considerations were necessary for cross-plane analysis because observations of the model through the plexiglass water tunnel walls from shallow angles yieided severely distorted images. Therefore, a mirror was installed in the tunnel so that the model could be viewed from a downstream perspective without distortion. The mirror was mounted at the base of the test section (approximately 1.5 body lengths from the base of the model) and tilted $45^{\circ}$ to the flow direction. The mirror was approximately 10 by $15 \mathrm{~cm}$ ( 4 by $6 \mathrm{in}$ ) and did not appear to have a significant effect on the flow field near the model.

\section{Flow Seeding}

Injected Fluorescent Dyes. Vegetable dyes (food coloring) are typically used during conventional operation of the water tunnel. However, for this experiment, it was necessary to use dyes that fluoresce under laser radiation. The three dyes evaluated in this study were fluorescein sodium, rhodamine B, and rhodamine $6 G$ (Table 1). The dyes were obtained in solid form, dissolved in water in measured concentrations, and injected through dye ports in the modeis. Initial experi- mentation with equal concentrations of dyes showed that the rhodamine dyes rapidly clouded the test section. For further comparison testing, the rhodamine dyes were mixed in concentrations by weight of $20,25,30,40,50$, and $60 \mathrm{mg} / 11 \mathrm{ter}$, while fluorescein was mixed in higher concentra. tions of $50,100,150,200,250$, and $300 \mathrm{mg} / 1$ iter. All of these dyes require care in handling, but the rhodamine dyes are suspected carcinogens and demand extreme caution in use, 5,6

Embedded Particulate Matter. Alternatively, flow can be visualized by illuminating particulate matter in the water tunnel water system. The LEV technique illuminates only those particles in the plane of light. Light reflected by the particles produces streaks on time-exposed photographic f $11 \mathrm{~m}$; these streaks can be measured and relative velocities determined. An advantage of using embedded particles is that the test model need not be fitted with separate tubing for the dye ports.

Experiments were conducted using different types of particulate matter (11sted in Table 1). Dirt and mineral deposits naturally present in the water were stirred up by increasing the flow rate in the water tunnel system. Other particulates were added to the water tunnel water system in measured amounts.

Models

Three models were used in this study (Fig. 4). One was a model of the space shuttle orbiter configuration having several orifices on the fuselage and wing upper surfaces from which dye could be emitted.

The second model was of the AV-8A Harrier configuration, equipped so that a controlled mass flow was sucked into the inlets (and a controlled flow was emitted from the exhaust nozzles) to simulate engine operation. Dye was mixed with the exhaust nozzle flow for flow visualization. The distance between the Harrier model and one of the test section walls was varied to simulate the effects of ground-plane proximity.

The third model, a circular body of revolution with an ogive forebody, a truncated base, and a circular disk behind, was used to study the flow characteristics associated with a trailing disk. Several external dye orifices were installed on the body surface forward of the base region.

Experimentation with model surface preparation included painting the model white, medium gray, or black with glossy and matte finishes (Table 1). Reference stations, marked on the model surface, were necessary to align the laser light sheet and to interpret photographs. Different methods for marking the reference stations (lead pencil, grease pencil, and colored tape) were compared.

Photographic Equipment

Color prints, slides, or video recordings were desired for documentation of flow visualization. 
Table 1 lists the various photographic techniques attempted. For prints and slides, a $35-\mathrm{mm}$ film camera was mounted on a tripod, and $f$ stops were varied from 3.5 to 1.2. Shutter speeds were sampled across the entire range of the camera, from $1 / 4$ to $1 / 1000 \mathrm{sec}$.

Experimentation with black-and-white film and slide film was limited because color prints were considered to be the most useful for documentation purposes. A drawback of slide film is that quality is sacrificed if prints are made from slides. It should be noted that the LEV photographs in this paper are reverse-image black-and-white reproductions of the original color pictures to improve quality for publication purposes.

It was important that all images were printed to actual object size to insure that reference marks for each test condition could be easily applied. Reference marks on the prints from reference station 1 were traced onto transparencies. These were laid on top of the prints taken from other laser reference stations at the same angle of attack. Bright spots on the prints showed where the dyes crossed the laser light sheet. These areas were charted, and trends were sketched. In this manner, vortex location, size, and burst point were determined. The flow patterns inside the vortices were also observed.

For video recording, two lenses ( $f 1.6$ and $f 1.4$ ) were compared.

\section{Results and Discussion}

The results of this study include development of test procedures that can be used to produce reliable results. Several specific aspects are discussed (summarized in Table 1). In addition, experiences with three examples that utilized the technique are discussed.

\section{Laser Power Setting}

Photographs were obtained for a test matrix of various power settings and dye concentrations. In the original test setup satisfactory results were obtained at a laser power setting of $750 \mathrm{~mW}$. In the modified setup, using direct laser light sheet projection, satisfactory results were obtained at $250 \mathrm{~mW}$.

\section{Flow Seeding}

Injected Fluorescent Dyes. Of the three dyes tested, fluorescein sodium (which fluoresces a bright green color) proved superior at the laser power settings used. It was determined that the preferred concentration of fluorescein in water is about $250 \mathrm{mg} / \mathrm{liter}$ ( $\mathrm{F} 1 \mathrm{~g} .5$ ). Although higher concentrations may slightiy increase the intensity of the dye, they also cause the water tunnel to more rapidly become saturated with dye, which hinders visibility and requires frequent drdinage of the tunnel.

Embedded Particulate Matter. Although it was easy to see the naturally occurring particulates with the eye, they did not reflect enough light to be recorded easily on film. Other types of particulate matter, including diatomaceous earth, glitter, and aluminum powder, were unsatisfactory because the particles clumped together and were difficult to mix into the water system. Later it was found that aluminum powder $(200$ mesh) worked well when a very small amount of liquid soap was added to the water to reduce surface tension, this method of using aluminum powder allowed excellent visualization of simple flow patterns.

The primary drawback of embedding particles in the flow field was the accumulation of particulate matter in the water tunnel system, this necessitated frequent drainage of the water and contributed to increased wear of the pump and filtering systems. In addition, particulate matter often accumulated on the models and was difficult to remove while the tunnel was operating. Results gained by this method are somewhat more difficult to interpret because the image is not as obvious as with the injected dye technique. However, the use of embedded particles eliminates the possibility of local flow field distortion caused by dye injection and prevents the ambiguous interpreta$t i o n$ of results owing to specific locations of dye ports. For these reasons, results obtained by the embedded particle method may be more valid.

\section{Model Preparation}

It was determined that models should not have a glossy surface, because visualization was degraded by significant laser light reflection outside the two-dimensional sheet. In addition, white models reflected too much light, and black models were difficult to mark with reference lines, therefore, a medium-gray color with a matte finish proved superior. Reference marks made with a grease pencil were quickly and easily drawn on the body of the model and could be changed with ease. Lead pencil marks were difficult to see, and colored tape did not adhere in the water tunnel flow.

\section{Photographic Techniques}

Photography proved to be one of the most difficult aspects of the study. The flow patterns were easily observed by the eye; however, the patterns were not sufficiently intense to be easily photographed. Long exposure times were not acceptable owing to the rapid motion of the flow. For color prints and slides of streamise flow patterns, a $35-\mathrm{mm}$ film camera, mounted on a tripod, with an f1.2 50-mm lens produced excellent photographs at shutter speeds of $1 / 125$ and $1 / 250 \mathrm{sec}$.

Flow patterns in the horizontal cross section were usually steady and could be photographed using slower shutter speeds. Good photographs were obtained using the $f 1.2$ lens at shutter speeds of $1 / 60$ and $1 / 80 \mathrm{sec}$.

Many kinds of $35-\mathrm{mm}$ color prirt films were compared. An ASA $1000 \mathrm{film}$ proved superior for this application. Films with lower ASA ratings were not sensitive enough to photograph at the low light 
levels of the test. Films with higher ASA ratings produced grainy prints.

For video recording, little difference was found between the f1.6, 50-mm lens and the fl.4, $50-m m$ lens. With the background lighting off, the fluorescing dyes were easily recorded on video tape, however, the model and other test apparatus were not visible. It was therefore necessary for reference purposes to have the background lighting on during some portion of the video recording.

\section{Sample Results}

The following review of specific studies using the three models demonstrates the results obtainable by employing the techniques and procedures previously discussed.

Space Shuttle Orbiter Model. The space shuttle orbiter model was chosen for evaluation because it has been the subject of well-documented flow visualization studies using conventional techniques. The orbiter model was painted a matte gray, and reference marks were drawn on the body. Streamwise and cross-plane laser reference stations were selected at $1.27-\mathrm{cm}(0.50-1 n)$ intervals on the wing (see Fig. 4).

A streamwise sheet of laser light was projected onto the model at stations 1 to 6 . At each angle of attack $\left(0^{\circ}, 5^{\circ}, 8^{\circ}, 10^{\circ}, 15^{\circ}, 20^{\circ}, 25^{\circ}\right.$, $30^{\circ}$, and $\left.40^{\circ}\right)$ a series of pictures, one at each laser station, were taken on the same roll of film. This procedure insured that the camera angle remained constant and that prints from the same film series could be overlayed. Overlaying prints proved neccessary because the reference marks were visible only on photographs of the most inboard laser stations. Figure 6 shows a series of photographs taken at an $8^{\circ}$ angle of attack with outlines of the orbiter superimposed to aid interpretation. Photographs of the cross-plane patterns were obtained in a similar manner. An example is shown in Fig. 7. Results from the LEV study correlated well with those from conventional water tunnel dye tests of the space shuttle model.

AV-8A Harrier Model. The second study was designed to evaluate ground effects during landing or takeoff of an AV-8A Harrier airplane. Figure 8 shows the results of this study (the dye paths of the flow field have been outlined graphically for clarity). The LEV technique results proved superior to conventional water tunnel test results on this model because more detall of the flow patterns could be observed. Another advantage was that relatively lower concentrations of dye were needed for the LEV technique (as opposed to conventional testing), which meant that longer test times were possible.
Trailing Disk Model. A final study was conducted to evaluate vortices trapped by a trailing disk behind the base of a cylinder. Figure 9 shows two photographs of the same test configuration with injected fluorescent dye (Fig. $9(a)$ ) and embedded particle (Fig. 9(b)) techniques. Both techniques provided excellent results.

\section{Concluding Remarks}

Results showed the laser enhanced visualization technique to be a useful flow analysis tool. The benefit of the technique is that a complex three-dimensional flow field can be reduced to two-dimensional images from which both qualitative and quantitative data can be extracted. The most significant drawbacks of this technique are the complication of the otherwise simple water tunnel test setup and the requirement for additional safety precautions.

Techniques and procedures are presented that produce reliable results. The procedures address installation considerations such as laser power levels, preferred dyes and concentrations, model preparation, and photographic documentation techniques. Potential users of the technique may find this information useful in system development. Generally the results show the following:

1. A laser power level of $750 \mathrm{~mW}$ was sufficient for all tests when mirrors were used for laser alignment. A laser power level of $250 \mathrm{~mW}$ was sufficient when the laser beam was projected through the cylindrical lens directly into the test section.

2. Dye concentrations of $250 \mathrm{mg}$ fluorescein sodium salt per liter of water provided good visualization when injected through the dye ports of the models.

3. Embedded particles (200 mesh aluminum powder) added to the water tunnel water system allowed for excellent visualization.

4. Model surfaces painted medium gray with a matte finish and grease-pencil reference marks were preferred.

5. An fl.2 aperture lens (shutter speeds of $1 / 125$ and $1 / 250 \mathrm{sec}$ for streamwise flow and $1 / 60$ and $1 / 80 \mathrm{sec}$ for cross-plane flow) and ASA 1000 color print film produced excellent $35-\mathrm{mm}$ color photographs.

6. Both fl.6 and fl.4 lenses worked well for video recording. 


\section{References}

lMerzkirch, Wolfgang, Flow Visualization, Academic Press, New York, 1974.

2McGregor, I., "The Vapour-Screen Method of Flow Visualization," J. Fluid Mech., vol. 11, pp. $481-511$.

${ }^{3}$ Staff, Aerodynamics Research Branch, "A Collection of Flow Visualization Techniques Used in the Aerodynamic Research Branch," NASA TM-85998, 1984.

4Saripal11, K.R., and Kroutıl, J.C., "A Novel Experimental Facility for Conducting Jet
Impingement Studies Related to VTOL Aircraft," AIAA-85-0052, 1985.

${ }^{5}$ Sax, Newton Irving, Dangerous Properties of Industrial Materials, 5 th ed., Van Nostrand Reinhold, New York, 1979.

6Lewis, R.J., and Tatkan, R.L. (eds.), Registry of Toxic Effects of Chemical Substances, National Institute for occupational Safety and Health, U. S. Dept. of Health and Human Services, Washington, D.C., 1979.

7Lorincz, Dale J., "Space Shuttle Orbiter Flow Visualization Study," NASA CR-163092, 1980.

Table 1 Test matrix considerations

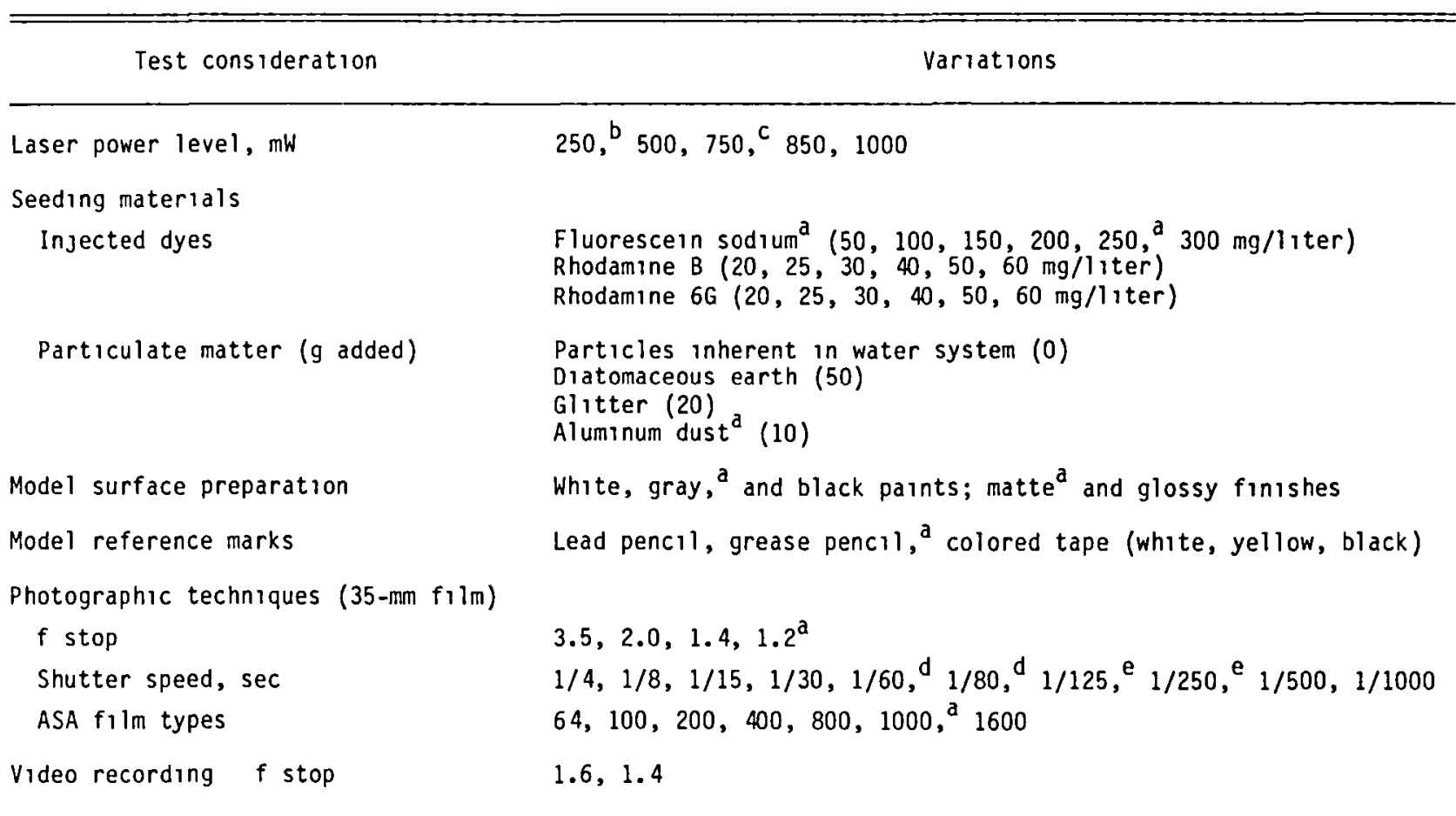

a variations that gave best results.

b Variation that gave best results with beam projected through cylindrical lens directly into water tunnel test section.

c Variation that gave best results when mirrors were used for laser alıgnment.

d Variations that gave best cross-plane visualization results.

e Variations that gave best streamwise visualization results. 

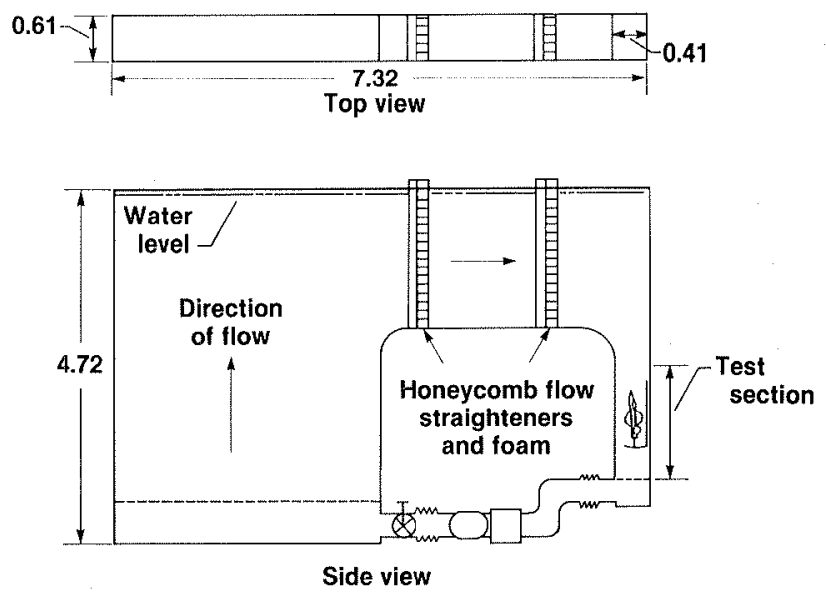

Water tunnel schematic (dimensions in meters)

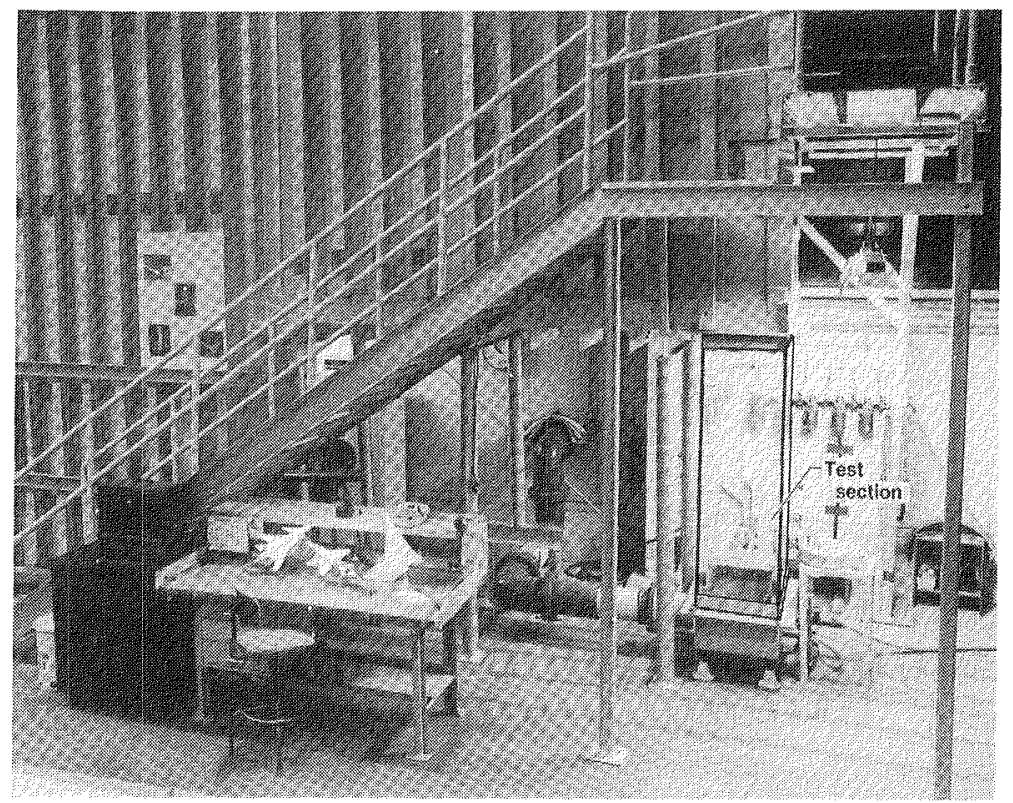

Photograph of water tunnel showing conventional test setup

Fig. 1 NASA Ames-Dryden flow visualization system. 


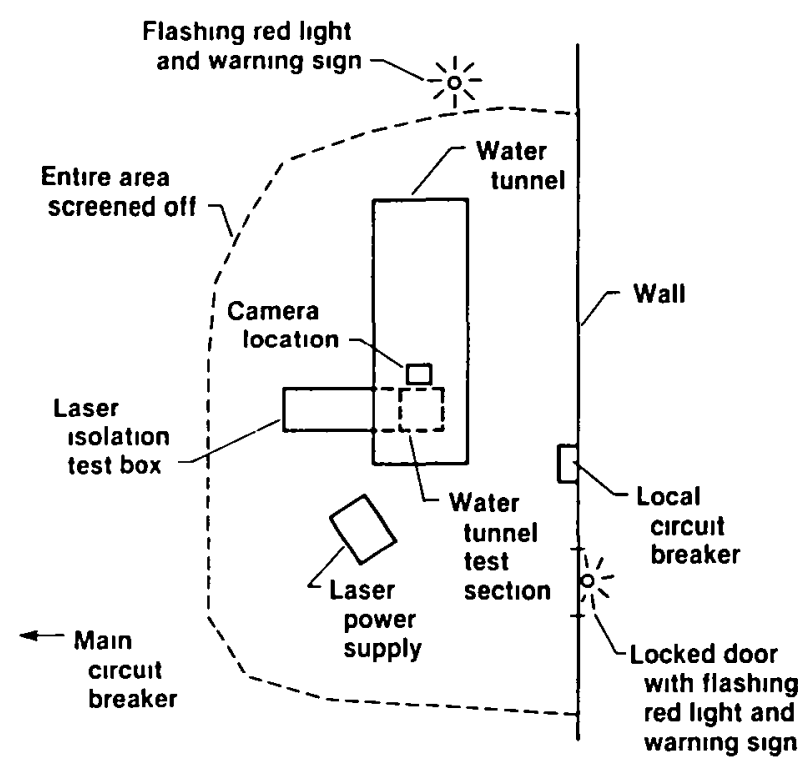

Top view of equipment layout

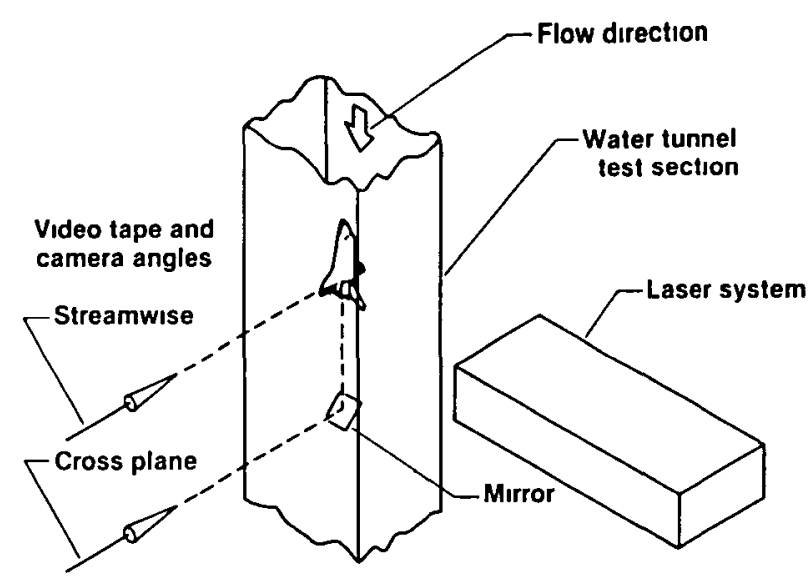

Test section arrangement

Fig. 2 Schematic of water tunnel test setup.

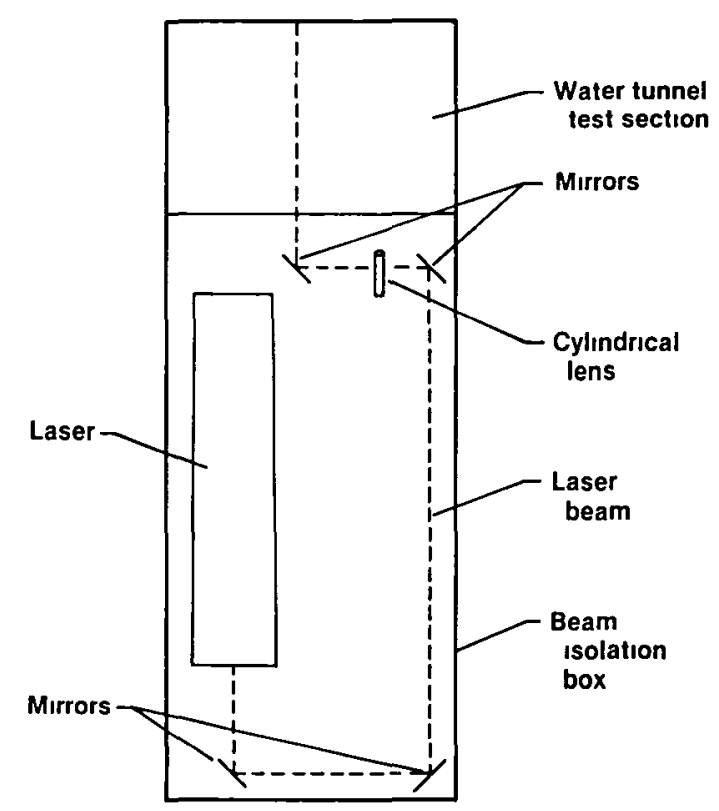

Fig. 3 Schematic of laser system. 


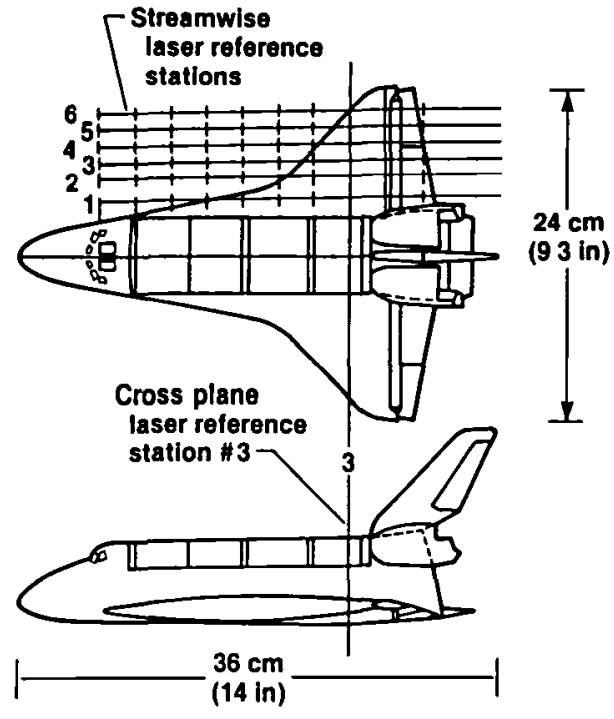

Space shuttle orbiter
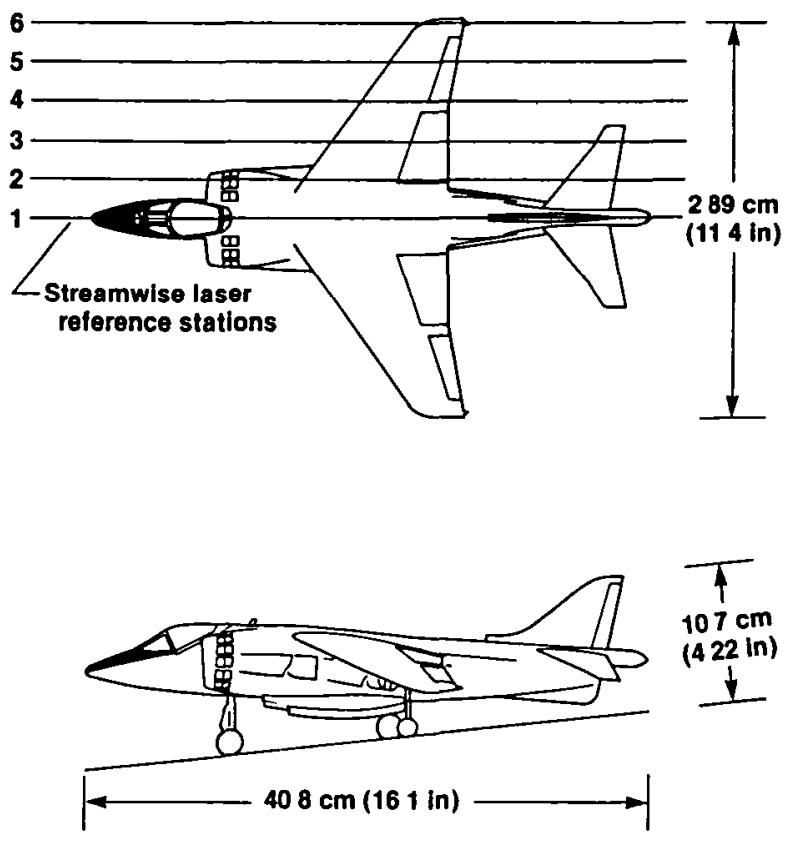

AV-8A Harrier

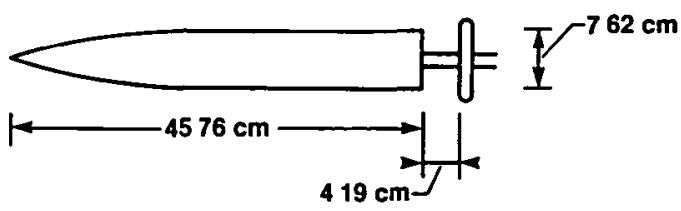

Trailing disk model

Fig. 4 Water tunnel modela. 


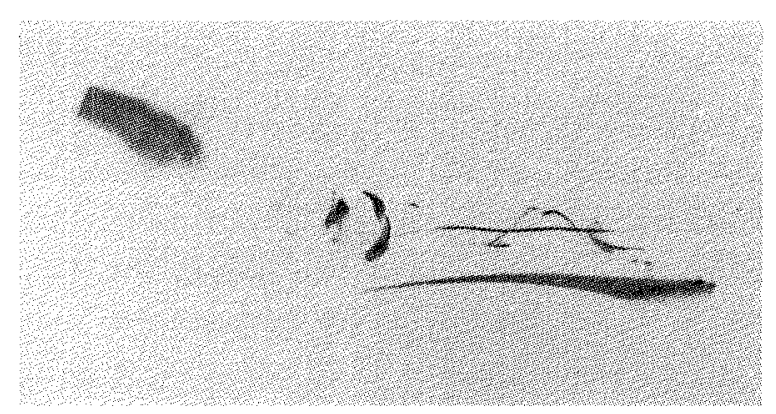

$50 \cdot 7 /$ liter

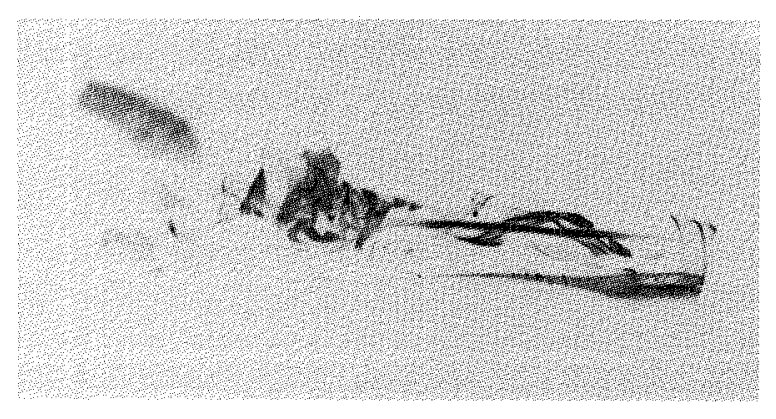

$150 \mathrm{mg} /$ liter

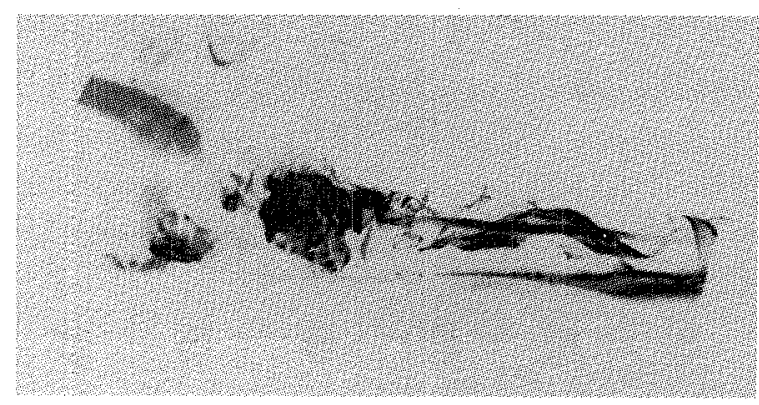

$250 \mathrm{mg} /$ liter

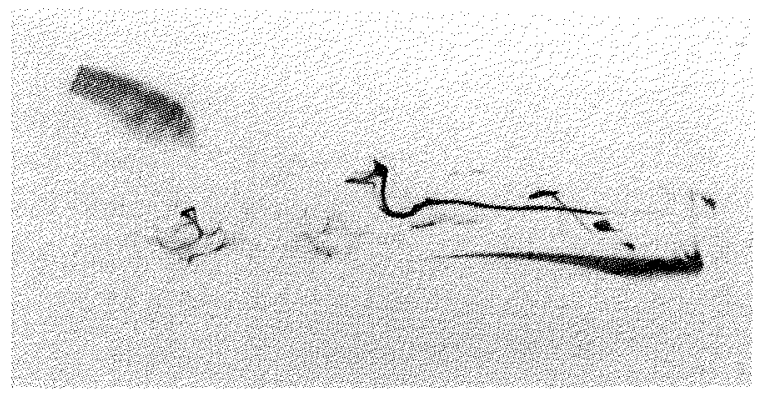

$100 \mathrm{mg} /$ liter

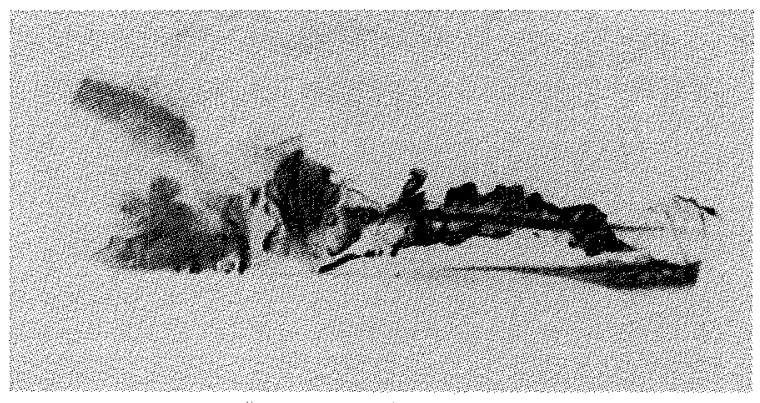

$200 \mathrm{mg} / \mathrm{liter}$

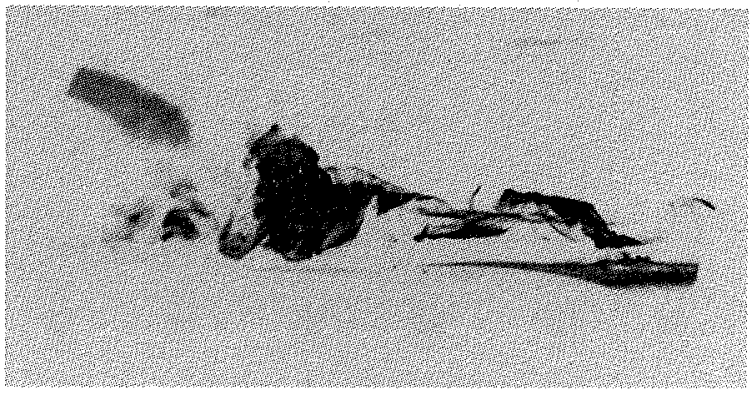

$300 \mathrm{mg} /$ liter

Fig. 5 Variation of flourescein sodium concentration, $750 \mathrm{~mW}$ laser power. 


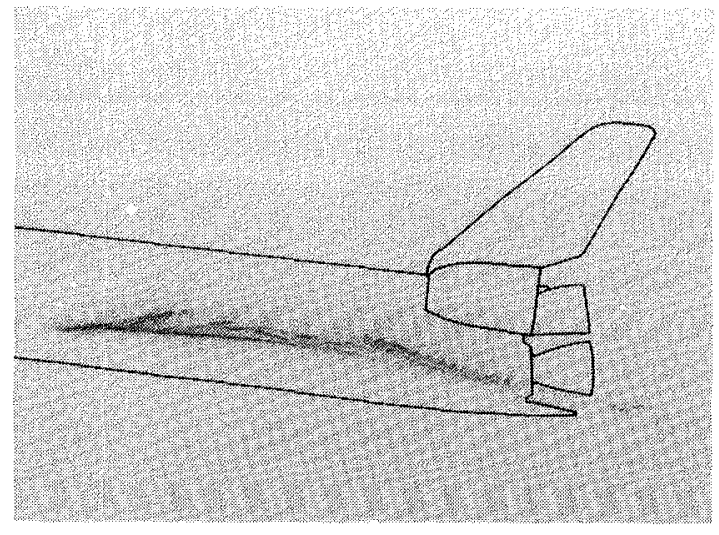

Station 1

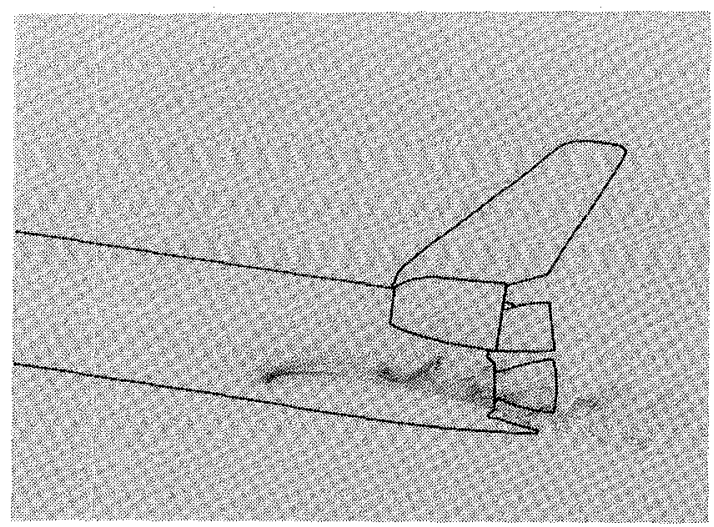

Station 3

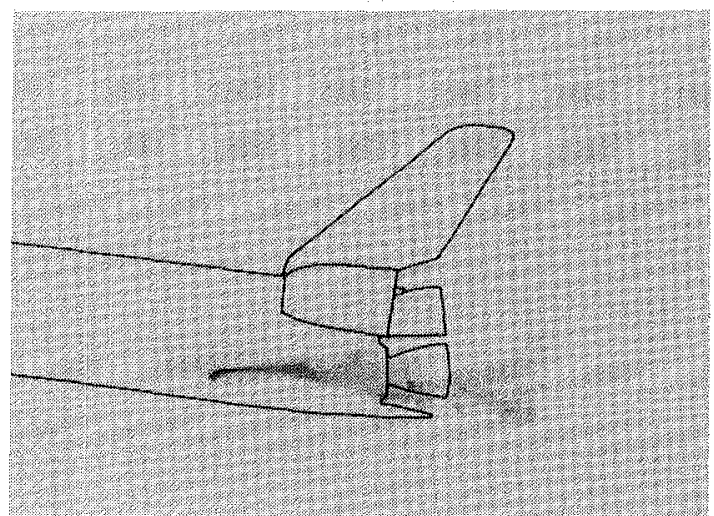

Station 5

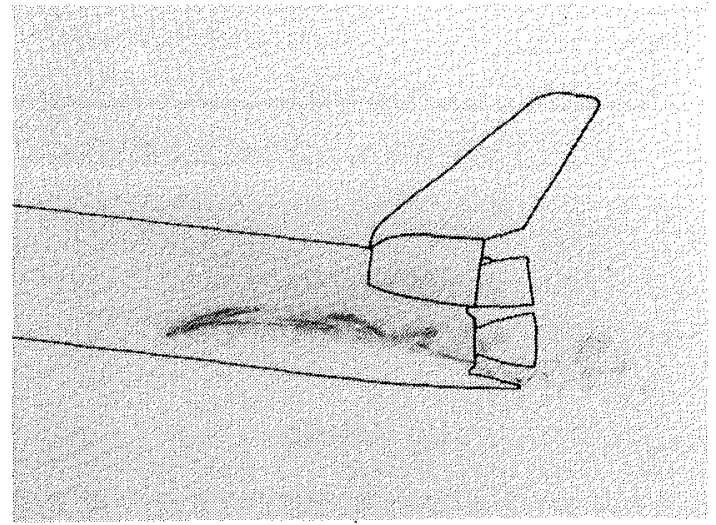

Station 2

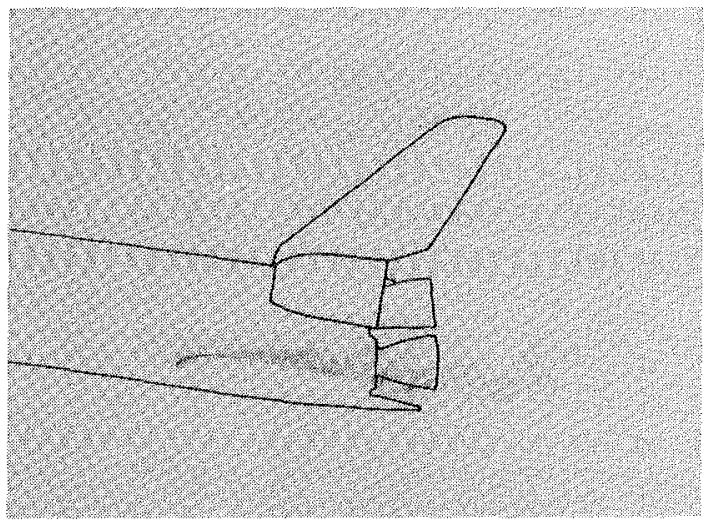

Station 4

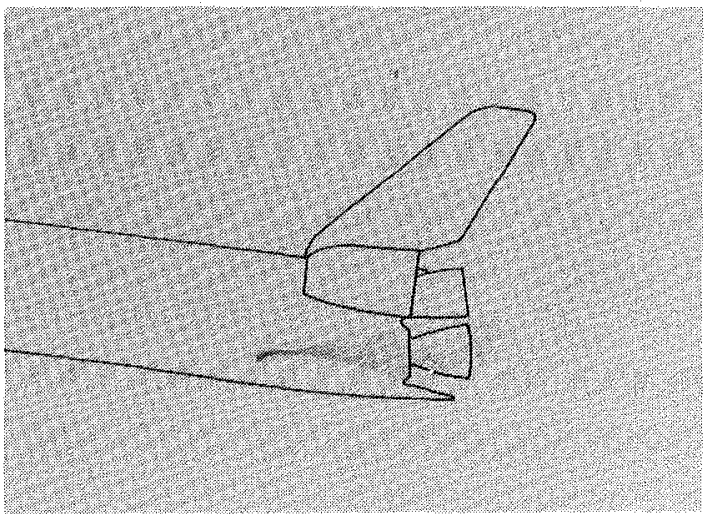

Station 6

Fig. 6 Examples of space shuttle orbiter results, streamwise laser stations, $8^{\circ}$ angle of attack. 

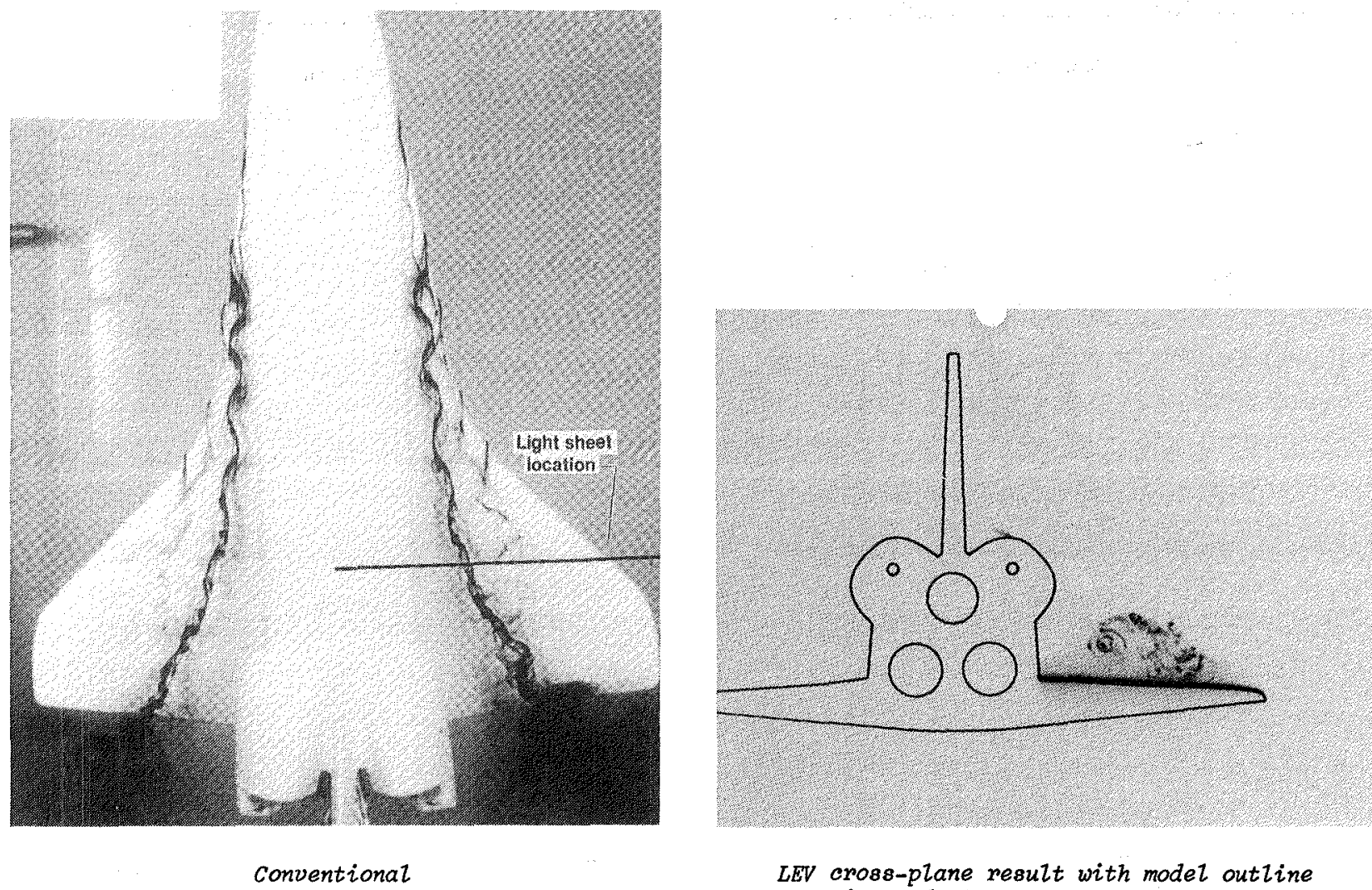

LEV cross-plane result with model outline superimposed

Fig. 7 Comparison of cross-plane LEV result and conventional water tunnel photograph. 


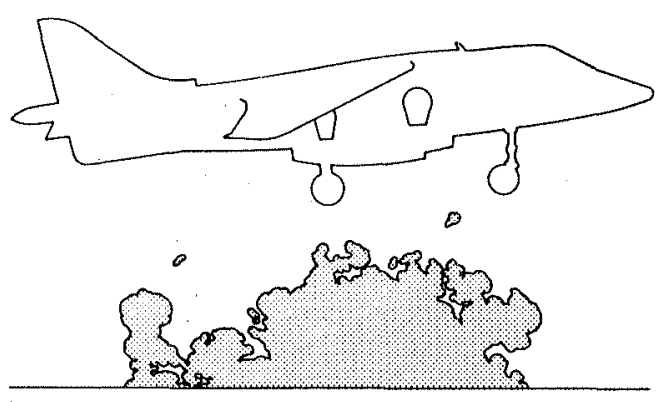

Station 1

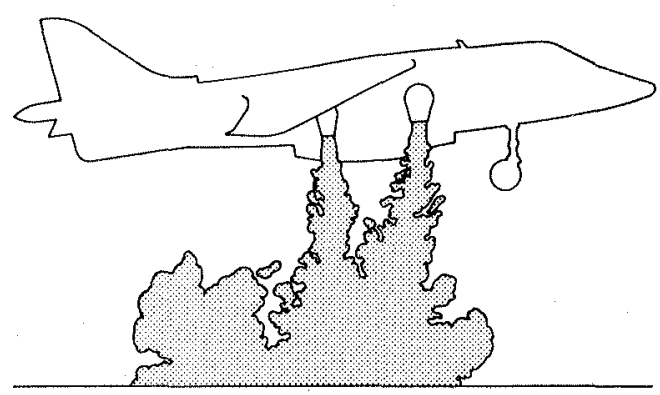

Station 3

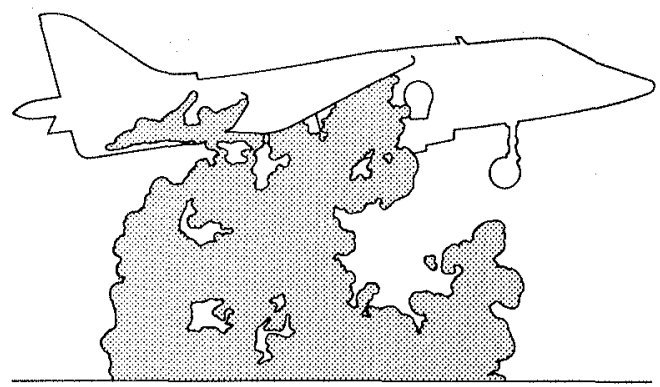

Station 5

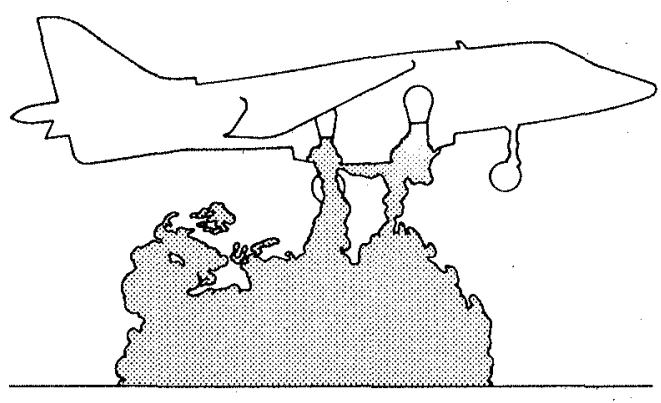

Station 2

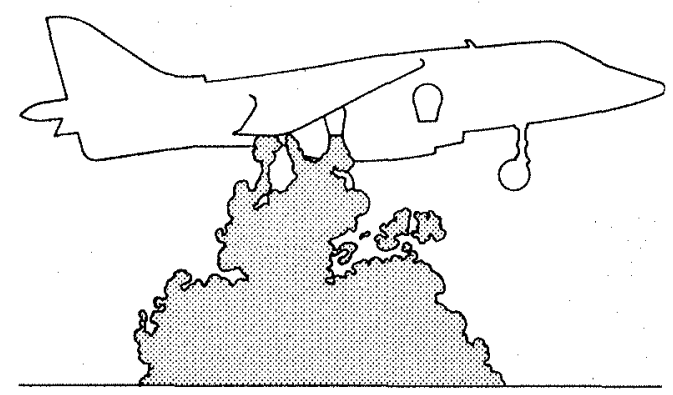

Station 4

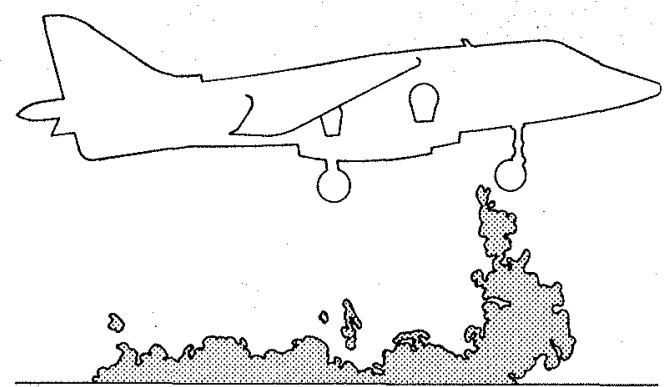

Station 6

Fig. 8 Example of Harrier model results showing ground effect in landing con-

figurations, 0.5 body lengths from

"ground" (water tunnel wall). 


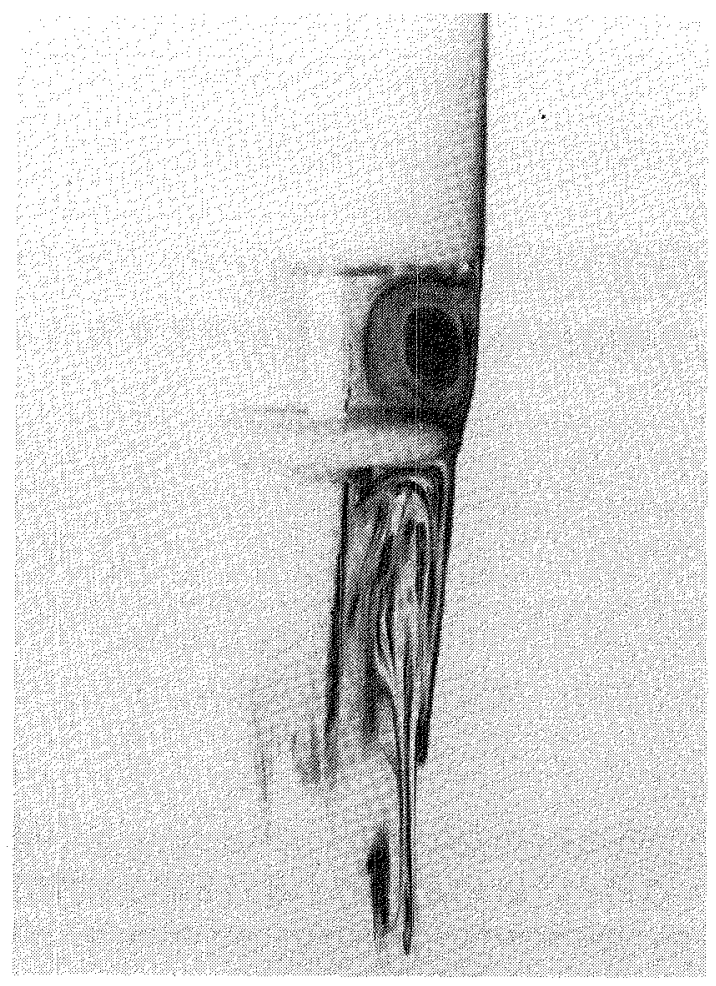

Injected dye technique

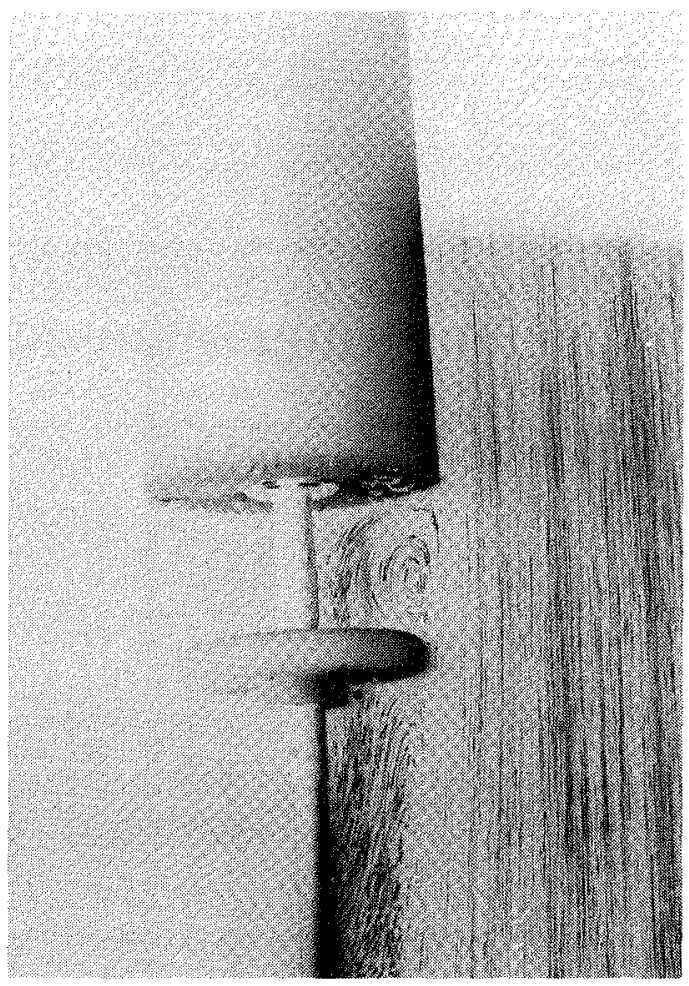

Embedded particulates technique

Fig. 9 Vortices trapped behind a cylinder by a trailing disk, comparing injected dye technique with embedded particulate matter technique. 


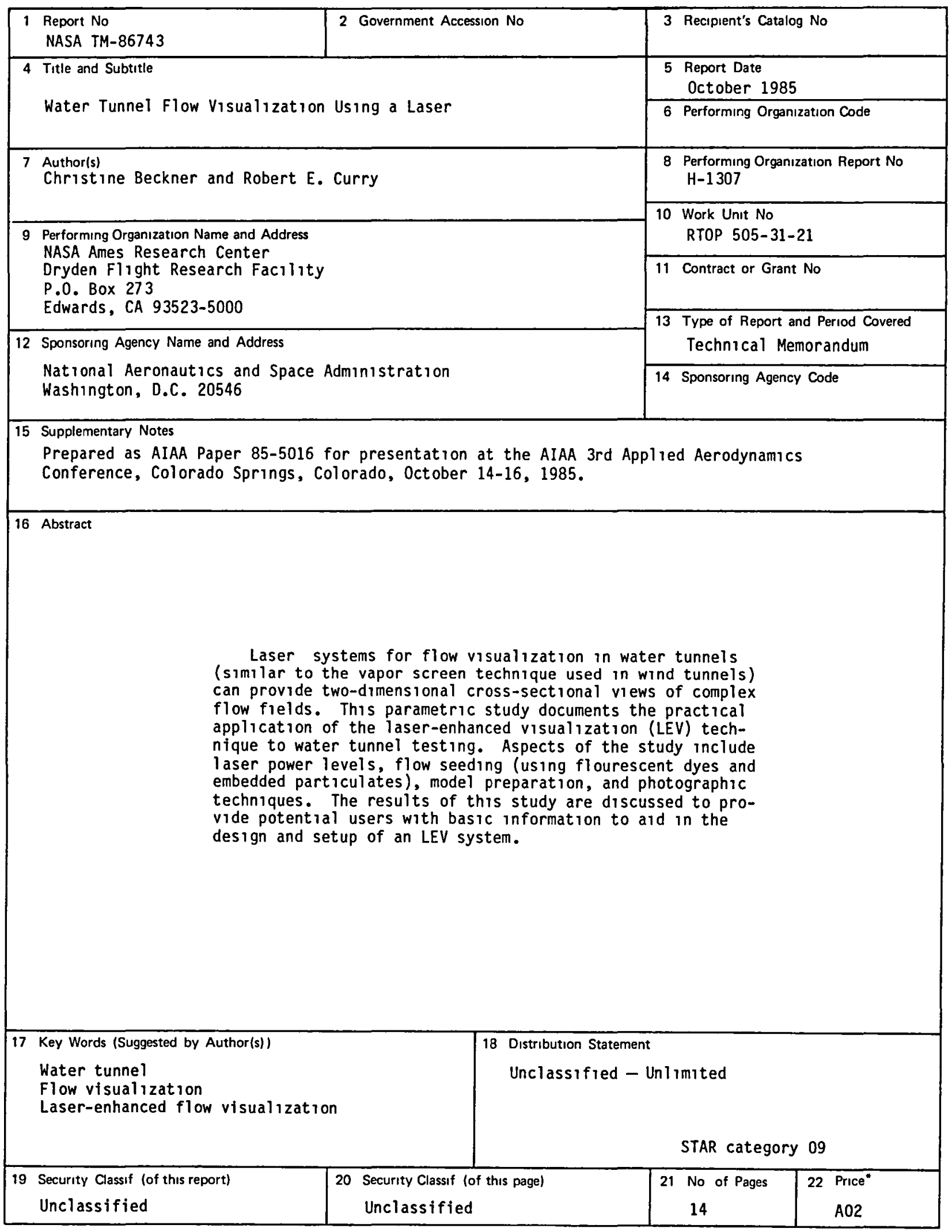

"For sale by the National Technical Information Service, springfield, Virginia 22161. 


\section{End of Document}

